\title{
Fístula perilinfática de causa traumática. Reporte de un caso pediátrico
}

Traumatic perilymphatic fistula. Report of a pediatric case

\author{
Dra. Elena Arias ${ }^{a}$ Dra. Daniela Cohen ${ }^{a}$ y Dra. Florencia Bianchia
}

\section{RESUMEN}

La fístula perilinfática de causa traumática es una patología poco habitual. En general, es causada por lápices, hisopos, hebillas de pelo y fósforos. Dentro de los síntomas más frecuentes, los pacientes pueden presentar hipoacusia y vértigo. Su diagnóstico requiere un examen físico completo que incluya otomicroscopía, audiometría y tomografía computada de ambos peñascos. El tratamiento depende de la sintomatología del paciente. En general, en un principio, es conservador, pero puede llegar a requerir cirugía. Se presenta un caso clínico de un niño de 6 años con fístula perilinfática secundaria a un traumatismo del oído izquierdo por un hisopo, que requirió tratamiento quirúrgico. Palabras clave:fístula, perilinfa, heridas y traumatismos, oídomedio.

\begin{abstract}
Traumatic perilymphatic fistula is an unusual pathology. Generally caused by pencils, swabs, hair buckles, and matches. Among the most frequent symptoms, patients can present hearing loss and vertigo.

Diagnosis requires a complete physical examination that includes otomicroscopy, audiometry and computed tomography of both boulders. Treatment depends on the patient's symptoms. In general, it is conservative at first, but may require surgery.

We present a clinical case of a 6-year-old boy with perilymphatic fistula secondary to left ear trauma due to swab, which required surgical treatment.
\end{abstract}

Key words: fistula, perilymph, wounds and injuries, middle ear.

http: / / dx.doi.org/10.5546/ aap.2021.e167

Cómo citar: Arias E, Cohen D, Bianchi F. Fístula perilinfática de causa traumática. Reporte de un caso pediátrico. Arch Argent Pediatr 2021;119(2):e167-e170.

a. División de Otorrinolaringología, Hospital General de Niños Pedro De Elizalde. Buenos Aires, Argentina.

Correspondencia:

Dra. Florencia Bianchi: florenciabianchi.orl@gmail.com

Financiamiento: Ninguno.

Conflicto de intereses: Ninguno que declarar.

Recibido: 18-6-2020

Aceptado: 25-9-2020

\section{INTRODUCCIÓN}

La fístula perilinfática (FPL) adquirida es uno de los mayores desafíos en la práctica otológica. No existe una técnica diagnóstica universalmente aceptada. ${ }^{1}$ Dentro de las causas más comunes de FPL, se incluyen el trauma penetrante, el barotrauma, la cirugía del oído medio y la fractura del hueso temporal. ${ }^{2}$

El daño de la cadena de huesecillos del oído medio provocado por traumatismos penetrantes es muy poco frecuente en la población pediátrica. La luxación del estribo con o sin apertura de la ventana oval ocurre, en general, por la introducción en el conducto auditivo externo (CAE) de un cuerpo extraño, frecuentemente, un hisopo o cotonete, que produce la perforación en la membrana timpánica $y$, si es muy profundo, daño en la cadena osicular. ${ }^{3}$ La fractura del estribo ocurre en alrededor de $1 / 3$ de las lesiones traumáticas osiculares. ${ }^{4}$

La FPL se asocia, por lo general, a mareos e hipoacusia. No existe consenso sobre su tratamiento debido al limitado número de casos reportados. ${ }^{5}$

\section{CASO CLÍNICO}

Niño de 6 años, sin antecedentes otológicos, que consultó por presentar otodinia y otorrea izquierda de 12 horas de evolución, posteriores a la introducción de un hisopo en el CAE. En la exploración, se constató una perforación amplia de la membrana timpánica y otorrea clara pulsátil. No refería hipoacusia ni síntomas vestibulares. Se realizó una audiometría tonal, la cual mostró una hipoacusia conductiva moderada en el oído izquierdo y fue normal en el oído derecho. Se indicaron pautas de alarma y reposo. Se citó en 24 horas.

En el control, se observó la misma imagen otomicroscópica. Debido a la sospecha de FPL, se solicitó una tomografía computada (TC) de ambos peñascos y una nueva audiometría. La TC mostró velamiento parcial de la caja timpánica y del mastoides del lado izquierdo. No se evidenció la alteración en la cadena de huesecillos ni imágenes compatibles con pneumolaberinto (Figura1). 
La audiometría reflejó una hipoacusia mixta en el oído izquierdo y fue normal para el oído derecho (Figura 2). Se decidió su internación con tratamiento médico.

Debido a la persistencia de salida de líquido claro a través de la perforación de la membrana timpánica y a la hipoacusia neurosensorial progresiva, al quinto día de internación, se decidió realizar la exploración quirúrgica. Durante el acto

FIgURA 1. Tomografía computada de ambos peñascos con ocupación parcial de la caja timpánica y mastoides izquierda por imagen de densidad de partes blandas. No impresionaba pneumolaberinto

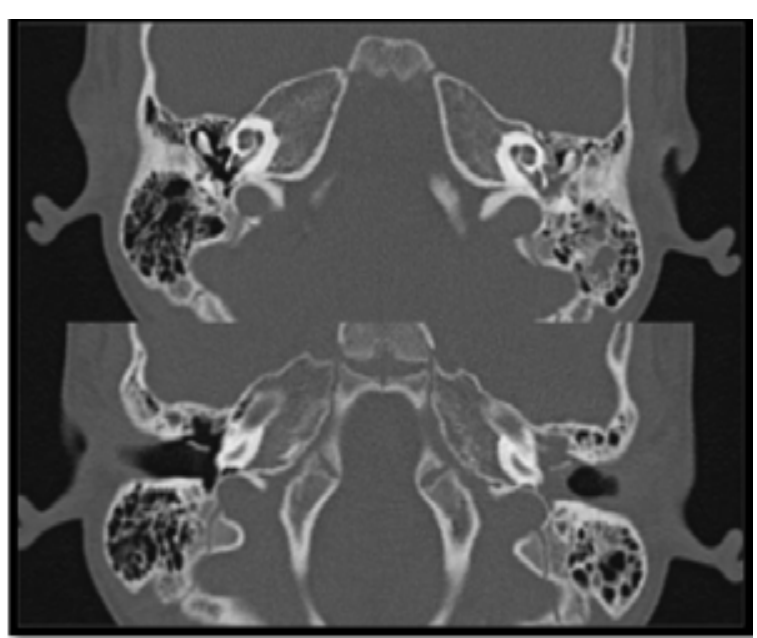

quirúrgico, se observó la mucosa inflamada, salida de líquido claro a nivel de la ventana oval; la cadena osicular se encontraba íntegra y móvil; el estribo no estaba luxado. Se colocó fascia para cubrir las ventanas oval y redonda, y, por encima, cola de fibrina (Beriplast ${ }^{\circledR}$ ) para sellarlas completamente (Figura 3). Se decidió no reparar la perforación timpánica para permitir el control posterior.
Figura 2. Audiometría tonal. Hipoacusia mixta de moderada a grave

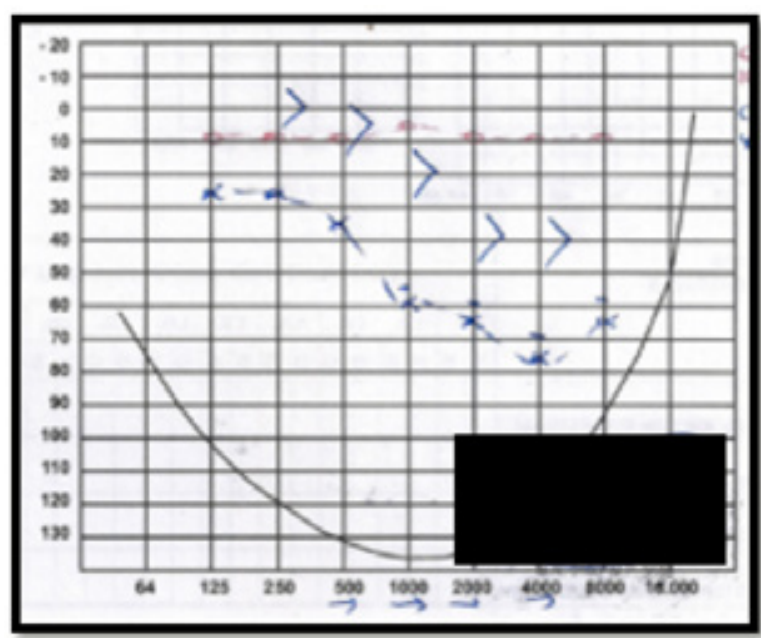

Figura 3. Imagen intraquirúrgica del oído izquierdo. A. $\star$ Apófisis corta del martillo. $\rightarrow$ Salida de líquido claro. B. Se colocó fascia (萧) para cubrir la ventana oval
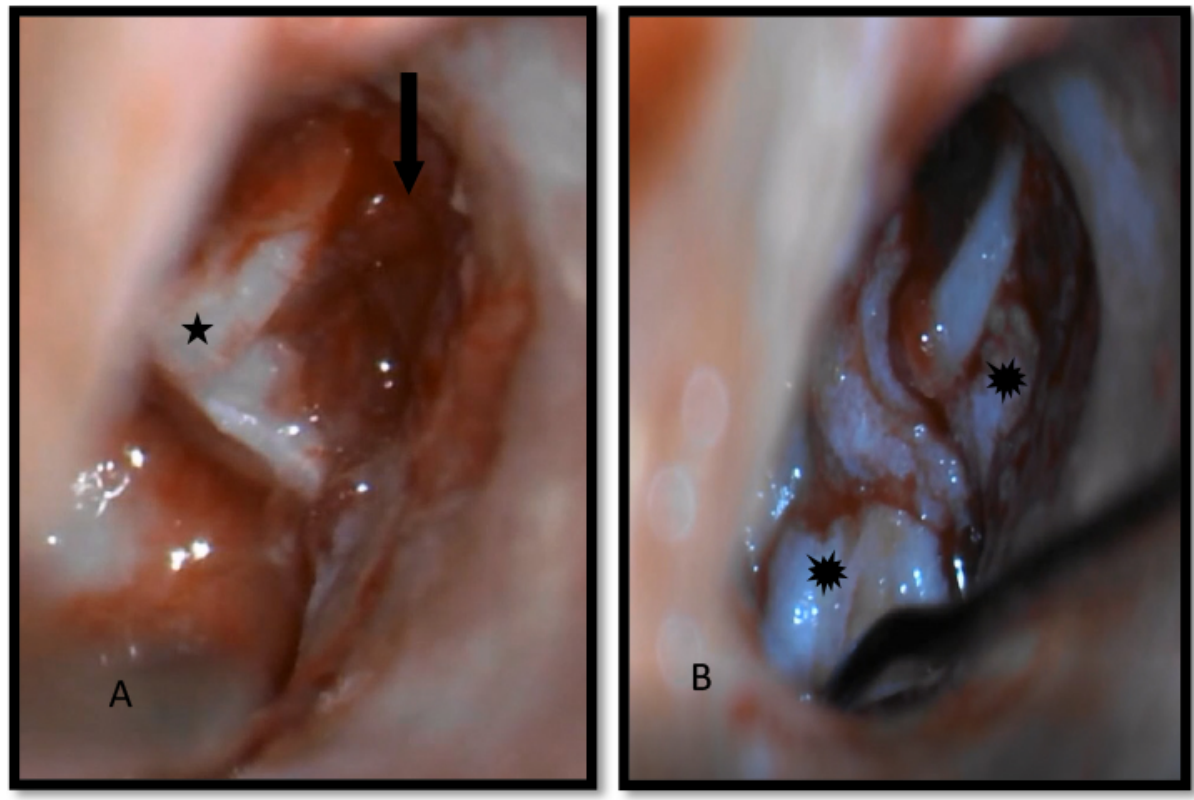
El paciente evolucionó favorablemente y se otorgó el egreso hospitalario a los 7 días de la cirugía. Al mes de seguimiento, presentó buena evolución clínica. Se observó una perforación pequeña en la región posterior, con oído seco y sin signos inflamatorios. La audiometría presentó una curva compatible con hipoacusia neurosensorial moderada izquierda (Figura 4), por lo que se sugirió la posterior selección de otoamplífono para el oído izquierdo.

\section{DISCUSIÓN}

Los traumas directos en la membrana timpánica u oído medio ocurren, mayormente, en los niños. Los instrumentos más comúnmente utilizados son lápices, hisopos, hebillas de pelo y fósforos.

La lesión del oído medio puede producir perforación de la membrana timpánica, hemorragia, dislocación y fractura de la cadena osicular, FPL desde la ventana oval o redonda, lesión de la cuerda del tímpano o parálisis facial. ${ }^{3}$ La FPL es una comunicación anormal entre el oído interno y el oído medio secundario a un daño en la ventana redonda o la ventana oval. En el caso de una dislocación estapediovestibular, la FPL es el resultado de una disrupción del ligamento anular o fractura de la platina. ${ }^{6}$

Los síntomas asociados a FPL tienden a aparecer rápidamente. El grado de pérdida auditiva varía de leve a profunda. ${ }^{7}$ Otros síntomas son el vértigo posicional agravado por la maniobra de Valsalva o el nistagmus inducido por presión sobre el trago. ${ }^{8}$

Figura 4. Audiometría tonal. Control al mes de la cirugía. Persistía la hipoacusia neurosensorial de moderada a grave

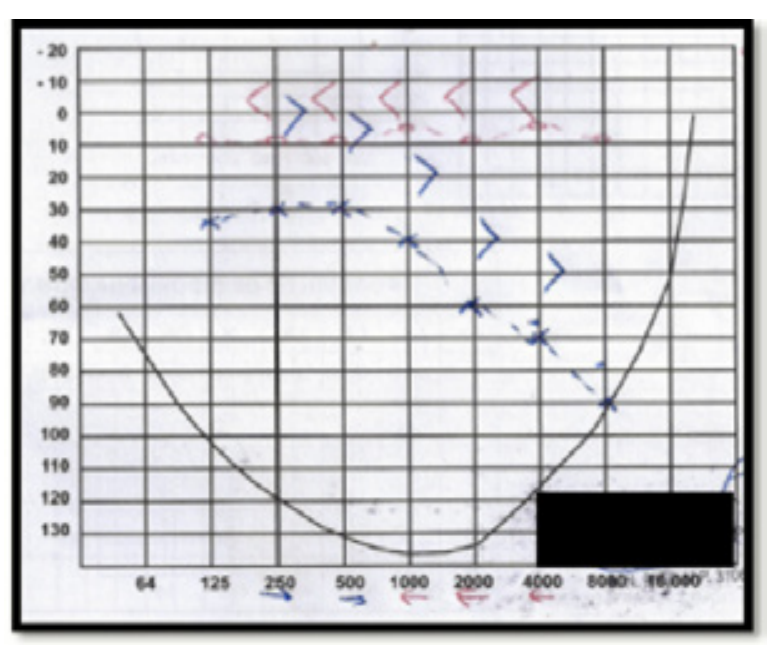

Si se sospecha FPL, se debe pedir una TC de peñascos de alta resolución para evaluar la presencia de pneumolaberinto. Este se detecta fácilmente cuando es de gran tamaño, pero, si es pequeño, puede ser un gran desafío hallarlo. ${ }^{2}$

Existen controversias sobre si, en caso de evidenciarse pneumolaberinto, es necesaria la exploración quirúrgica o no. Sin embargo, la presencia de aire en el oído interno no significa, necesariamente, una fístula activa, ya que esta puede haberse cerrado y haber dejado aire en el interior del laberinto. ${ }^{6}$

Pueden hallarse diferentes posturas en cuanto a la conducta. Algunos autores proponen el tratamiento conservador en el caso de pneumolaberinto y/o luxación del estribo.

En una de serie de 3 casos de pneumolaberinto por trauma penetrante (sumado a la revisión bibliográfica de otros 48 casos), Hidaka ${ }^{5}$ observó que no había diferencia significativa en la mejoría de la audición entre el grupo que había recibido tratamiento quirúrgico con respecto al que no lo había recibido. El tratamiento conservador puede continuar si no existe progresión de la hipoacusia o persistencia de síntomas vestibulares. En caso contrario, se debe realizar una cirugía exploradora. ${ }^{5}$

Tsubota ${ }^{9}$ describió el caso clínico de un paciente de 20 años que presentó un traumatismo por hisopo. Este, a pesar del tratamiento médico, evolucionó con hipoacusia progresiva y vértigo, por lo que requirió tratamiento quirúrgico. La cirugía consistió en reposicionar el estribo en la ventana oval, colocar fascia temporal y sellar con cola de fibrina.

Hay autores que proponen que, en el caso de FPL por trauma penetrante, primero, se debe realizar el tratamiento conservador con timpanotomía exploradora temprana (1-2 semanas) y cierre de fístula en el caso de aumento de síntomas vestibulares o hipoacusia neurosensorial. En general, luego de este procedimiento, los síntomas vestibulares mejoran, pero los efectos sobre la hipoacusia neurosensorial son inconstantes. ${ }^{3}$

Según Tsubota, el pronóstico de los síntomas vestibulares es bueno en los casos de pneumolaberinto, pero el pronóstico de los niveles auditivos es peor. A su vez, este autor propone 3 factores predictivos de la mejoría auditiva, basándose en series de casos reportados en la bibliografía:

1. El tiempo desde el traumatismo hasta la cirugía (en los pacientes en los que la cirugía 
había sido realizada antes de los 14 días, se vio mayor recuperación de la audición con respecto a aquellos en los que se había demorado más tiempo).

2. El nivel de conducción ósea en la audiometría (los pacientes que tenían niveles de conducción por vía ósea mayores de $65 \mathrm{~dB}$ al inicio de la enfermedad tuvieron peores resultados auditivos que los que presentaban niveles menores de $65 \mathrm{~dB}$ ).

3. La lesión del estribo. ${ }^{9}$ Con respecto a este último punto, hay diferentes posturas. Por ejemplo, Hatano ${ }^{10}$ propone que, en caso de luxación del estribo en el vestíbulo, el tratamiento dependa de cada paciente.

Es importante evaluar (con el fin de definir el plan quirúrgico) si el estribo está deprimido en el vestíbulo. Si lo está, cuán profundo se encuentra y si presenta o no fractura. Cuando el estribo está profundamente deprimido en el vestíbulo, el reposicionamiento quirúrgico aumenta el riesgo de causar daño en el oído interno, lo que origina resultados desfavorables en la audición posoperatoria. De lo contrario, si el estribo permanece en el vestíbulo, pueden generarse bridas alrededor de este, ocupar el espacio vestibular y causar un daño tardío en el oído interno. En el caso en que el estribo está levemente deprimido en el vestíbulo y la superestructura puede observarse en la ventana oval, entonces, el huesecillo debe ser removido. Sin embargo, debido al escaso número de casos descritos en la literatura, no hay un protocolo de tratamiento cuando el estribo está profundamente deprimido en el vestíbulo. ${ }^{10}$

En nuestro paciente, en una primera instancia, se consideró el tratamiento conservador, pero, al persistir la salida de líquido claro a través de la membrana timpánica (lo que indicaba que la FPL permanecía activa) y presentar una hipoacusia neurosensorial progresiva, se decidió realizar el tratamiento quirúrgico. Evolucionó, luego de la cirugía, con mejoría de la imagen otomicroscópica y estabilización de la hipoacusia.
En los casos de pneumolaberinto, el pronóstico de la audición es peor que el de los síntomas vestibulares. ${ }^{8}$ La FPL puede ocurrir como consecuencia del traumatismo de la cadena osicular y ventana oval. En ocasiones, se observa, en la TC, un signo patognomónico, como el pneumolaberinto. No hay un protocolo establecido para el tratamiento. En principio, se recomienda el seguimiento audiométrico y la evaluación de síntomas vestibulares. En el caso de presentar hipoacusia neurosensorial progresiva o aparición de síntomas vestibulares, se sugiere la cirugía.

\section{REFERENCIAS}

1. Nishiike S, Hyo $\mathrm{Y}$, Fukishima H. Stapediovestibular dislocation with pneumolabyrinth. J Laryngol Otol. 2008; 122(4):419-21.

2. Kita A, Kim I, Ishiyama G, Ishiyama A. Perilymphatic fistula after penetrating ear trauma. Clin Pract Cases Emerg Med. 2019; 3(2):115-8.

3. Van Hoecke H, Calus L, Dhooge I. Middle ear damages. B-Ent. 2016; Suppl 26(1):173-83.

4. Snelling JD, Bennett A, Wilson P, Wickstead M. Unusual Middle-Ear Mischief: Trans-Tympanic trauma from a hair grip resulting in ossicular, facial nerve and oval window disruption. J Laryngol Otol. 2006; 120(9):793-5.

5. Hidaka H, Miyazaki M, Kawase T, Kobayashi T. Traumatic Pneumolabyrinth: air location and hearing outcome. Otol Neurotol. 20121; 33(2):123-31.

6. Bogaerts M, Waterval J, Van Dinther J, Somers T, Et Al. Treatment of traumatic stapediovestibular luxation: case report with the introduction of a new technique and review of literature. Otol Neurotol. 2014; 35(4):582-8.

7. Nakashima T, Matsuda K, Okuda T, Tono T, et al. Late pneumolabyrinth may beinduced by old penetrating injury: possibility of undiagnosed posttraumatic perilymphatic fistula. Case Rep Otolaryngol. 2015; 2015:506484.

8. Achache M, Sanjuan Puchol M, Santini L, Lafont B, et al. Late pneumolabyrinth after undiagnosed posttraumatic perilymphatic fistula. Case reportillustrating the importance of systematic emergency management. Eur Ann Otorhinolaryngol Head Neck Dis. 2013; 130(5):283-7.

9. Tsubota M, Shojaku H, Watanabe Y. Prognosis of inner ear function in pneumolabyrinth: case report and literature review. Am J Otolaryngol. 2009; 30(6):423-6.

10. Hatano A, Rikitake M, Komori M, Irie T, et al. Traumatic perilymphatic fistula with the luxation of the stapes into the vestibule. Auris Nasus Larynz. 2009; 36(4):474-8. 